\title{
Erratum to: Investigating the Use of Social Media Technologies by Adults with Autism Spectrum Disorder in Saudi Arabia
}

\author{
Alaa Mashat ${ }^{(\bowtie)}$, Mike Wald, and Sarah Parsons \\ University of Southampton, Southampton, UK \\ \{a.mashat,mw, s.j.parsons\}@soton.ac.uk
}

\section{Erratum to: \\ Chapter 22: M. Antona and C. Stephanidis (Eds.) Universal Access in Human-Computer Interaction DOI: $10.1007 / 978-3-319-40238-3 \_22$}

In Table 1 on page 229 of the original version of the paper the last 5 rows were missing. The correct table is shown below.

Table 1. Participants' uses of social media

\begin{tabular}{|c|c|c|c|c|c|}
\hline Participant & Gender & Age & Devices & Used SN & $\begin{array}{l}\text { Created } \\
\text { SN for } \\
\text { the study }\end{array}$ \\
\hline P1 & Male & 30 & Ipad (shared with his sister) & None & Instagram \\
\hline $\mathrm{P} 2$ & Male & 23 & $\begin{array}{l}\text { Smartphone } \\
\text { ipad } \\
\text { PC } \\
\text { Laptop }\end{array}$ & $\begin{array}{l}\text { WhatsApp, (Facebook } \\
\text { and Twitter, could not } \\
\text { access it) }\end{array}$ & Instagram \\
\hline P3 & Male & 22 & $\begin{array}{l}\text { Smartphone Galaxy 5S } \\
\text { laptop (broken) }\end{array}$ & WhatsApp, Snapchat & Instagram \\
\hline $\mathrm{P} 4$ & Male & 19 & iphone $5 \mathrm{~S}$ & $\begin{array}{l}\text { WhatsApp, Snapchat, } \\
\text { Instagram Facebook, } \\
\text { Twitter, Keek, Path, BB } \\
\text { messenger }\end{array}$ & None \\
\hline $\mathrm{Pb} 5$ & Male & 23 & ipad 2 & WhatsApp & None \\
\hline $\mathrm{Pb} 6$ & Male & 16 & ipod & None & None \\
\hline
\end{tabular}

The updated original online version for this chapter can be found at 10.1007/978-3-319-40238-3_22 
Table 1. (continued)

\begin{tabular}{l|l|l|l|l|l}
\hline Participant & Gender & Age & Devices & Used SN & $\begin{array}{l}\text { Created } \\
\text { SN for } \\
\text { the study }\end{array}$ \\
\hline $\mathrm{Pb} 7$ & Male & 25 & $\begin{array}{l}\text { Galaxy smartphone and uses } \\
\text { his sister's iphone 6 }\end{array}$ & None & None \\
\hline $\mathrm{Pb} 8$ & Female & 19 & ipad 2 & None & None \\
\hline $\mathrm{Pb} 9$ & Female & 15 & $\begin{array}{l}\text { Does not have her own } \\
\text { device }\end{array}$ & None & None \\
\hline $\mathrm{Pb} 10$ & Female & 15 & $\begin{array}{l}\text { xbox, playstation3, } \\
\text { playstation4, ipod, iphone, } \\
\text { wii, wii U, PC, laptop, } \\
\text { Graphic drawing tablet }\end{array}$ & YouTube, Tumbler & None \\
\hline $\mathrm{Pb} 11$ & Male & 28 & $\begin{array}{l}\text { Samsung Galaxy } \\
\text { smartphone, Toshiba Laptop }\end{array}$ & None & Instagram \\
\hline $\mathrm{Pb} 12$ & Male & 15 & $\begin{array}{l}\text { Does not have his own } \\
\text { device }\end{array}$ & None & None \\
\hline $\mathrm{Pb} 13$ & Male & 18 & $\begin{array}{l}\text { Lenovo Smartphone, Tablet } \\
\text { (being repaired) }\end{array}$ & $\begin{array}{l}\text { WhatsApp, (Instagram } \\
\text { and Facebook, could not } \\
\text { access it) }\end{array}$ & None \\
\hline
\end{tabular}

